\section{HAKONE SHOKUBOTSU.}

(Dai 34 gö no tsusukki.)

\section{Epipactios}

gigantea. Hook.

San-chi:-Hakone.

Nihon mei:-Suzu-ran.

\section{Aceras}

angustifolia, Lindl. var. longicruris, Miq.

San-chi:-Jigoku; Futago-yama.

Nihon mei:-Mukago-sō.

\section{Mabenaria}

sagittifera, Reich. fil.

San-chi:-Hakone.

Nihon mei:-Aoyayi-sō.

(Plantanthera) Yatabei, Max.

San-chi:-Hakone.

Nihon mei:-Yamasagi-sō.

(Platanthera) oreades, Fr. et Sav. var. brachycentron, Fr. et Sav.

San-chi:-Hakone (Savatier shi).

Nihan mei:-

(Plantanthera) tipuloides, Lindl. var.

Ussuriensis, Regel. et Maack.

San-chi:-Hakone (Savatier shi).

Nihon mei:-Ōyamasagi-sō.

\section{Chondradenia}

Yatabei, Max:

San-chi:-Futagoyana

Nihon mei:-Kamome ran?

\section{HAMODORACAE.}

\section{Ophiopogon}

Japonicus, Gawl

San-chi:-Dōkan.no-taira; 'Tōnosawá.

Nihon mei:-Janohige. $\gamma$. Wallichianus, Maxim.

San-chi:-Hakone.

Nihon mei:-̄oba-janohige.

\section{IRIDE $A E$.}

\section{Iris}

Japonica, 'Thunb.

San-chi:-Hakone.

Nihon mei:-Shaga.

Sibirica, T.

San-chi:-Hakone.

Nihon mei :-Ayama.

\section{Belanicancla}

Chinensis, Adans.

San-chi:-Hakore.

Nihon mei:-Hiōgi.

AMARYLLIDEX.

\section{Lycoris}

sanguinea, Maxim.

San-chi:-Sengokubara mura.

Nihon mei :-Kitsune-no-kamisori.

\section{DIOSCOREACEA.}

\section{Dioscores}

sätiva, Miq.

San chi:-Hakone.

Nihon mei:-Naga-dokore.

quinqueloba, Thunb.

San-chi:-Ōsawa. (Makino Tomitarō shi saishū).

Nihon mei:-Kikuba-dokoro.

tenuipes, Fr. et Sav.

San-chi:-Hakone-jiku-moyorı. (Makino 'Tomitarō shi sāishū).

Nihon mei:-

sativa, $\mathrm{I}_{\text {. }}$

San-chi:-Moto Hakore moyorı (Makino Tomitarō shi saishū).

Nihon mei:-Maruba-ilokoro. 
Japonica, Thunb.

San-chi:-Hakone-jiku moyori. (Makino 'Tomitarō shi sai shūi).

Nihon mei:-Yamanoimo.

\section{Srailax}

herbacea, I. var. Nipponica, Maxim.

San-chi:-Hakone; Ōtome-tōge.

Nihon mei:-Siode.

Higoensis, Miq.

San-chi:-Hakone (Makino 'J'omitarō shi).

Nihon mei:-

Sieboldi, Miq.

San-chi :-Hakone (Maximowicz shi).

Nihon mei :-Yamakashī.

China, Is.

San-chi :-Dōkan-no-taira kimbō.

Nihon mei :-Sarutori-jbara.

\section{Polygonatum}

vulgare, Desf.

San-chi:-Hakone (Thunberg shi).

Nilon mei:-Amadokoro.

giganteum, Dietr. $\gamma$. falcatum, Maxim.

San-chi:-Miyanosita (Bisset. shi saishü).

Nilun mei:-Hosoba-ōsei.

$\delta$. macranthum, maxim.

San-chi:-Hakone (Maximowicz shi).

Nihon mei:- $\overline{\mathrm{O}}$-narukoyuri.

\section{Hemerocallis}

fulva, $I_{\text {. }}$.

San-chi:-Hakone.

Nihon mei:-Yabu-kanzō.

\section{Funkia}

Sieboldiana, Hook.

San-chi:-Ōsawa.

Nihon mei:-Tō-gibōshi. var. longipes, Fr. et Sav.

San-chi:- $\bar{O}$ sawa.

Nihon mei:-Iwa-gibōshi.

lancifolia, Spreng.

San-chi:-Hakone.

Nihon mei:-Mizu-gibōshi.

\section{Alliugn}

Japonicum, Reg.

San-chi:-Hakone.

Nihon mei:-Yama-rakkyō.

Senescens, L. var. Serotina, Regel.

San-chi:-Hakone (Savatier shi).

Nihon mei:-

\section{Seilla}

Japonica, Baker.

San-chi:-Sengokuhara-mura.

Nihon mei :-Tsurubo.

\section{Tilium}

Maximowiczii, Regel.

San-chi:-Hakone.

Nihon mei--Ko-oni-yuri.

auratum, Lindl.

San-chi:-Hakone.

Nihon mei:-Ryori-yuri.

cordifolium, Thunb.

San-chi:-Hakone.

Nihon mei: Uba-yuri.

\section{Metanarihecium}

luteo-viride, Maxim.

San-chi:-Hakone.

Nihon mei:-Nogiran.

\section{Tofieldia}

nuda, Maxim,

San-chi:-Hakone (Savatier shi). Nihon mei :-Hana-zekishō.

( Ika jigö.) 